\title{
CHAPTER ONE FINITE SOCIAL SPACE AND THE EVOLUTION OF HUMAN SOCIAL BEHAVIOUR
}

\author{
ROBERT FOLEY AND PHYLLIS LEE
}

\section{SOCIAL EVOLUTION: A PROBLEM OF DEFINITION}

Within the traditions of archaeology and anthropology, a common set of oppositions is that between the biological and the social. The putative contrast between what is biological and what is social has led to explanations for patterns of variation in human behaviour and in prehistory which are seen as competitive or mutually exclusive. The growth of 'social' archaeology, for example, might easily be seen as an alternative to the more ecological approaches that dominated in previous decades. If we are to investigate biologically based approaches to the evolution of social behaviour, it is clear that against this background it is necessary to find a way through a number of conflicting concepts and definitions of the term 'social'.

The most extreme interpretation of the term 'social' is that it is the antithesis of what is biological. Things are either social or they are genetic and biological. This view, indeed, conflates the term 'biological' to mean the same as genetic, a common fallacy within the social sciences (Ingold 1990). This is a strongly anthropological and non-evolutionary perspective, which implies that sociality is a unique part of the human world. It ignores the fact that the capacity for social behaviour is predicated upon physical and biochemical and indeed genetic characteristics, the widespread occurrence of social behaviour among other species, and the apparent trend towards marked sociality that can be found among the primates.

A second view is that the term 'social' refers specifically to the presence of elaborate cognitive skills. Among humans the variation in social behaviour is exemplified by cultural diversity, which in turn implies a strong association between the social and the cognitive and symbolic systems that humans have developed. While the previous view took the term 'social' away from that which is biological, within this second framework the concept of 'social' is removed from actual behaviour and focused on the cognitive capacities that generate behaviour. This view can be extended to include the idea that sociality is dependent upon the capacity for symbolic systems of thought and a sense of self-consciousness. While cognition is clearly an important element of any social behaviour, it is not the totality even if, as some have argued, the evolution of greater intelligence is related to social complexity (e.g. Humphrey 1976). The social world is actually played out in the realm of behaviour.

This perspective on the social is strongly linked to the general notion of culture in 
anthropology; indeed, the terms 'culture' and 'social' are often used in an almost interchangeable manner. Culture, it could be argued, is concerned with the transmission of ideas and information through non-biological means, and as such it is transmitted largely through social channels. This view conflates the cultural capacities of humans with the tendency towards social life: to be social requires non-biological means of passing on information, and culture in turn requires individuals to live in social groups. There is thus a positive feedback between the two (Foley 1991, McGrew 1992).

From the zoological end we have other notions of what is meant by the term 'social'. For some biologists it simply means group living - that is, a social species is any one where individuals do not lead solitary lives, and all groups can be considered as social groups. Clearly this greatly extends the meaning of the term, and takes the problem of social evolution away from anything very special to include an enormous variety of biological problems, from clonal colonies of bacteria to shoals of fish. While there may be advantages in this view, the generality is too great to have much explanatory power; while many species may aggregate into groups of conspecifics, it is not necessarily the case that their interactions are in any meaningful sense of the word social. It is probably the case that the majority of social species live in groups, but it is not the case that all groups are indeed social.

It is obvious that any attempt to model or explain social evolution is highly dependent upon the way in which the term 'social' is used. In adopting those that are more specifically anthropological and which exclude biology, the power of any evolutionary approach is greatly reduced. Expanding the definition to all associations loses the focus on that which makes humans, and other primates, unique.

The solution we have adopted here is that formulated by Hinde $(1976,1983)$. Sociality is seen not as a top-down system imposed by the characteristics of the group as a whole, but rather as an emergent property derived from the interactions between individuals. Social groups thus refer to groups where associations are maintained over time and space, where the individuals are consistently interactive, where individual recognition of others can be found, and where associations are patterned by familiarity and genetic relatedness. The interactions themselves typically can be classified into a number of simple categories. When these categories of interaction are patterned over time, repeated between individuals, and both the context and the content of the interaction are replicated, then social relationships emerge from simple rules of interaction. These relationships can also be patterned, maintain a stable context and content, and produce a form of social structure which is unique to that cluster of individuals. Such an approach does not ignore the perceptual, communicative or cognitive elements inherent in transactions; rather, in non-linguistic species it provides a basis for definition and comparison (Lee 1992). It also stresses the distinction between the mechanisms by which sociality is maintained (for example, cognition, behavioural cues, etc.) and the actual behaviour of being social. Indeed, Hinde's approach (see Hinde 1983) emphasizes the dialectic between the individual, the relationship and the emergent system. Again, the nature of the relationships, which themselves influence interactions, and so on, provides for a non-linear approach to understanding the complexity of sociality, at least for species with 'maintained sociality' (Lee, in press).

This means that the task of investigating the evolution of human sociality consists of 
determining the nature and extent of the repeated interactions that could have occurred in ancestral groups of hominids, the ecological, social and demographic conditions under which these may have changed, and the consequences of the interactions and subsequent sets of relationships for the emergence of human characteristics and behaviour. To this end we have developed a model of finite social space (Foley and Lee 1989, Lee, in press) that allows us to attempt to track the pathways of hominid social evolution and to explore its Darwinian basis.

\section{FINITE SOCIAL SPACE: A MODEL OF SOCIAL OPTIONS}

The central element of this model is that the range of options for social interactions, relationships and structure are finite and can be specified. This is in direct opposition to the widely held anthropological view that the range of social variation is infinite and therefore that reconstructing past states of sociality is an impossibility. In contrast, we have argued that because there are clear finite limits to what is possible socially, and because the conditions under which these variants may occur are relatively narrowly constrained, it is possible by a process of elimination to focus on the social options available for humans and their ancestors.

The assumptions of the model are predicated upon a biological constraint typical of mammalian and, indeed, many other species (Trivers 1972, Emlen and Oring 1977). This constraint can be simply stated as the differential costs of reproduction to males and females; male gamete production is relatively inexpensive in terms of time and energy, and a male's reproductive potential is limited by his access to potential mates. Females, on the other hand, produce more energetically costly gametes, bear the energy and time costs of internal gestation and subsequent lactation. Female reproduction is thus largely limited by time and energy, through her condition and nutritional intake. In using this basic premise, we are placing the biology of reproduction - the physiological, environmental and time costs - at the core of potential sociality, and have drawn on Wrangham's (1980) original premise for primate sociality His framework argues that females need to ensure access to sufficient energy for reproduction, and the way in which energy (in the form of food resources) is distributed in the environment will ultimately determine the distribution of females in the environment, while male distributions will follow those of the females and take into account the number of other males also attempting to maximize access to reproductive females.

In terms of the model, other reasons for aggregating such as to avoid predation may influence group size. However, the fundamental structure of the group and its maintenance over time will depend on the options available to females to distribute themselves through a habitat in order to maximize foraging and nutritional intakes, thus ensuring reproduction and infant survival. Any female therefore can be limited to three simple options in their distribution with respect to other females. First females can be alone, without associating with other females. Second, females can associate with other females; here there are again only two options. Either they can be together with their other female kin, or they can be with females who are unrelated. This produces three 'social states' for females (Figure 1.1). 
Males have the same options with respect to distributions of the same sex. Males can either be alone, relative to other males, with male kin or with male non-kin. Three options are thus available for male distributions. These three social states can be superimposed across the sexes, so that a total of nine permanently social states emerge, which combine the male and female options. Of course, there is no need for males and females to be continuously in association, and indeed among many mammals, as well as for some of the non-human primates, males and females live separately outside the mating periods. If the male-female associations can be categorized as stable or transitory, then the number of social options increases to eighteen (Figure 1.1). Among the primates, however, with one exception, all these species are among the strepsirhines (lemurs, galagos and lorises) where female receptivity is seasonal, of short duration within an oestrus period and where male-female co-residence provides few reproductive, foraging or anti-predator benefits to either sex. Among the higher primates, males and females are continuously co-resident, since

\section{Male distribution state}

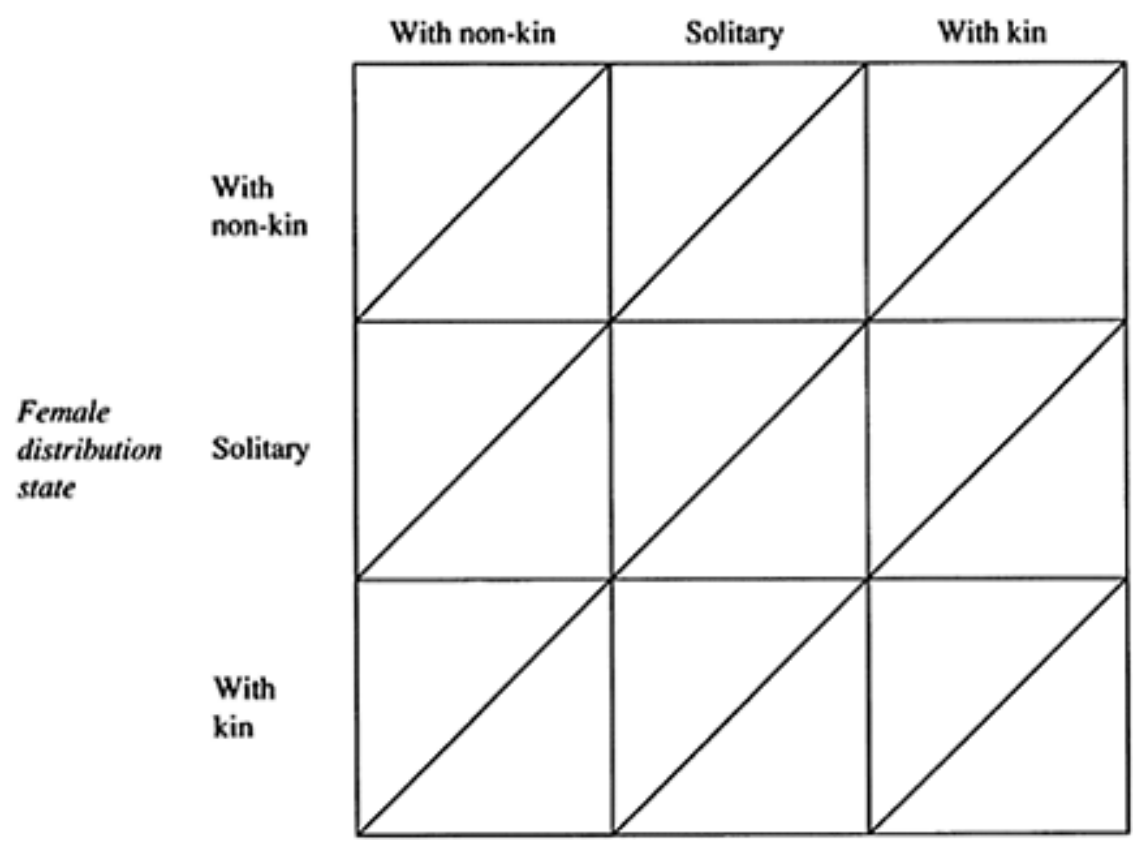

Figure 1.1 Model of finite social space. The vertical axis shows how females associate with other females (solitary, with kin or with non-kin), and the horizontal axis shows how males similarly associate with other males. Interactions between the these two intra-sexual associational strategies provide the basis for social structure. A further dimension is added by allowing male-female reproductive associations to be either stable or transitory. The combinations yield a basic number of eighteen social structures derived from individual associations and 
these females have menstrual cycles of regular duration, are receptive for longer parts of the cycle, and show less marked seasonality to their cycling periods in any year. Under such conditions, male-female distributions have stabilized into the small range of options mentioned above.

These distribution states are, of course, purely hypothetical social states. They say little about the size of the group or the nature of the interactions which maintain the group. As a reduction of complexity to its bare minimum, they nonetheless highlight some interesting features. First, some social states are either rare or non-existent, while some are extremely common (Figure 1.2) across a range of mammalian species. For the most part, mammalian same-sex distributions appear to confirm the principles on which the model is based. Second, through comparisons between

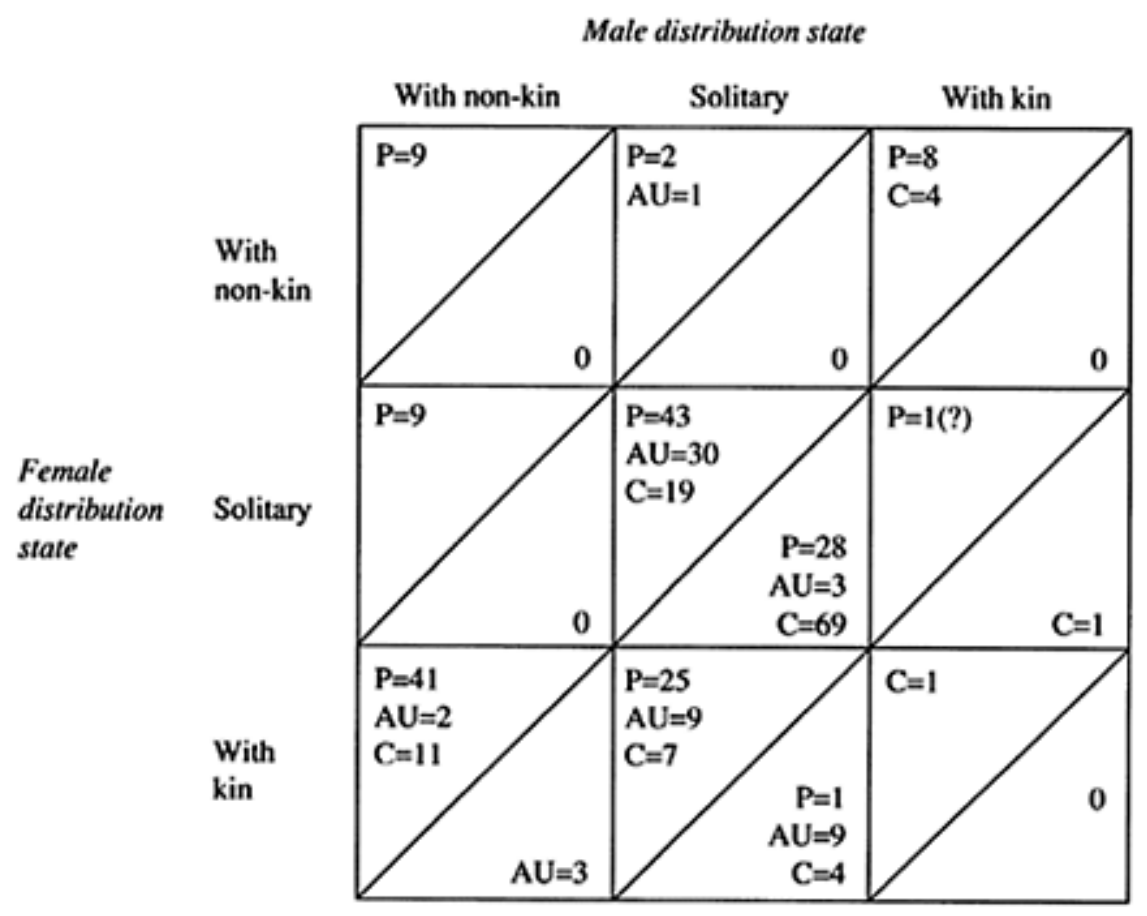

Figure 1.2 Frequency of social systems according to the finite social space model for a sample of three mammalian groups: Primates $(\mathrm{P})$; African ungulates (AU) and Carnivores (C). The most frequent pattern is female kin-bonded groups $(113 / 46.5 \%)$, followed by monogamy $(92 / 37.9 \%)$. Male kin-bonding is the rarest structure $(14 / 5.8 \%)$. (Modified from Lee, in press)

species with known ecological conditions, it becomes possible to specify the ecological conditions whereby some of these states will arise and stabilize. Finally, the model allows 
for complexity to be superimposed, in that the distributions define limits within which social complexity can flourish, and allows us to define the starting point for constructions of complexity.

\section{FINITE SOCIAL SPACE AND SOCIAL EVOLUTION}

This brief description of the finite social space model allows us to draw a number of inferences about the nature of social evolution, as well as to indicate a number of possible pathways for exploring the history of human sociality.

The main point of the model is that social variability in terms of male and female distributions is severely limited; within this model there are a maximum of eighteen possibilities. These have been extended by adding a number of more finely divided categories, such as recognizing the existence of inter-generational associations within kin groups (Foley and Lee 1989), or that inter-sex associations may vary more subtly than between just transient and stable. Even with these taken into account, though, the number of options remains limited.

Second, not all social systems are equally close to each other in evolutionary terms. For example, chimpanzees have a social system based on male residence and female dispersal - i.e. they are male kin-bonded - and inter-sexual relationships are both stable over the medium term while transient on a day-to-day basis. Gorillas, where there is a single male harem system, differ in two ways from chimpanzees - they are not male kinbonded (males are solitary with respect to other males) and male-female relationships are stable. Orangutans, on the other hand, differ from gorillas and chimps by only one state change-relative to chimps in terms of male kin-bonding and relative to gorillas in terms of the stability of male-female relationships. Examining the 'distance' between social states allows us first to make estimates about the probability of various social states being ancestral to others and second about the probability of moving along particular evolutionary trajectories to certain end states.

The notion of evolutionary pathways is important, as one element of any evolutionary analysis is determining what has evolved from what. While this is relatively straightforward in principle at least in many evolutionary systems (for example, anatomy), its basis in social evolution is less well established. Put simply, it is generally accepted among evolutionary biologists that any evolutionary change is the outcome of the interaction between novel selective pressures and the existing structure or behaviour. Evolutionary change is therefore not just a product of a new environment, but of how the existing structures interact with the new environment. The same novel selective pressures can produce entirely different evolutionary responses because the initial conditions differ. For example, both apes and monkeys were faced with the selective challenge of declining forests and expanding grasslands in Africa in the later Miocene, but their responses were divergent: certain apes (hominids) became bipedal as this was the most cost-effective response in the context of a climbing, partially brachiating primate, while monkeys (baboons) simply transformed their arboreal quadrupedalism into terrestrial quadrupedalism. The same principles will hold for social evolution, and therefore in order to know how hominids evolved socially in response to new ecological and social 
conditions it is necessary to determine the ancestral states involved. Phylogeny and phylogenetic context are therefore of considerable importance and power within the finite social space model.

Third, while social states are partially constrained by phylogeny, they are also strongly influenced by immediate costs and benefits, and therefore can be analysed in terms of the principles of behavioural ecology. The 'bottom up' approach to sociality adopted here means that the occurrence of particular social structures will be the outcome of the costs and benefits of the following: (1) females forming groups with specific female compositions; (2) males forming more or less stable relationships with females; and (3) males and females tolerating the presence of other males. The costs and benefits of each of these will be a function of the resources available and utilized. Again, these costs and benefits will be dependent upon both the existing life-history parameters of the individuals and the external resources.

The significance of these last points lies in the logical conclusion that there is bound to be an ecological and energetic element for social evolution (as well as for setting the initial conditions for sociality), and that this will operate in ways that are dependent upon phylogenetic context. With this in mind we will now examine the phylogeny of human sociality and then its ecological context.

\section{PHYLOGENETIC CONTEXT OF HUMAN SOCIALITY}

The interpretation of the phylogenetic context of Homo sapiens has undergone considerable change recently Two factors are mainly responsible: first, the increasingly unambiguous evidence from genetics and molecular biology that humans and the African apes are more closely related to each other than either is to the Asian orangutan; and second, the evidence that human evolution consists of a series of adaptive radiations and multiple taxa rather than a single unilinear trend. While there is considerable controversy beyond these points, both about terminology and details, nonetheless these two lines of evidence provide us with the basis for considering the phylogenetic context of the hominids and the way in which social behaviour fits into it (see also Ghiglieri 1987, Wrangham 1987, Foley 1989).

The living Old World anthropoids or Catarrhini are divided into two super-families, the Hominoidea and the Cercopithecoidea. The former comprise the apes and humans, the latter the monkeys (including macaques, baboons, mangabeys, guenons and the leafeating colobines and langurs). This evolutionary divide is seen in a number of physical, ecological and behavioural differences. There is also a marked difference in social strategy. Cercopithecoids are primarily characterized by male dispersal between groups and female residence. Where kin-based alliances occur, they are based on matrilines. In terms of the model, males are either solitary with respect to other males or co-resident with non-kin males. The exceptions to this pattern are found among the Colobinae, of which the red colobus (C. badius) is the best studied example (Struhsaker and Leland 1987). The red colobus is unusual in forming groups with male kin and general female dispersal. Among some langurs, both sexes can disperse from the natal group to form new social units lacking any kinship. However, this is also the state that is reflected in 
monogamy, and is relatively simple to produce under the terms of the model.

In contrast to the typical female-kin residence pattern found among most cercopithecoid primates, among the Hominoidea stable female-kin residence is unknown. Both males and females can disperse, removing any potential degree of kin association, as in the monogamous gibbon (Hylobates spp.) and the solitary orangutan (Pongo pygmaeus). Among gorillas, again, females disperse prior to reproduction as do the majority of males, although some males remain resident with their fathers and these can ultimately inherit the harem (Harcourt and Stewart 1987). Among the common chimpanzee (Pan troglodytes) females generally disperse, while males remain co-resident with their male kin and form strong kin-based co-operative alliances (Goodall 1986). The situation among the pygmy chimpanzee or bonobo (P. paniscus) is interesting, in that females probably disperse and males remain co-resident, but the alliance structure seems to be concentrated on the male-female relationships rather than focused within the male kin units (Kano 1992).

This social distinction is striking. Using the model to define probable state changes from a solitary state to one of either male or female kin distributions produces a pattern where viable female kin units are far more likely to arise though a small number of state shifts than are male kin units (Foley and Lee 1989, Lee, in press). While the cercopithecoids display the mammalian conservative condition of female residence, the apes show the more derived form (Figure 1.3). Hanging the various social states on the most probable cladogram for the catarrhines allows us to identify the most probable social characteristics at the various branching points that have occurred in the evolution of the Hominoidea. Although homoplasies and reversals, as well as far greater diversity among the hominoids in the past is likely to complicate the picture, the following appears to be the most parsimonious interpretation of the social changes that have occurred during the evolution of the hominoids.

1 At the branching of the cercopithecoids and hominoids female residence seems to have lapsed. This is most likely to have occurred in the context of monogamy, in which both sexes disperse. This pattern is retained among the gibbons.

2 Increasing body size among the apes appears to have led to greater male tenure in harems, resulting in females dispersing to join successful males, thus re-emphasizing the break from female residence. This pattern is found in different ways among the gorillas (orthodox harem) and the orangutan (solitary or 'exploded' harem).

3 Under some circumstances males began to remain in their natal groups. The proposed mechanism for this is that with the break-up of the lush Miocene forested environments females began to range more widely for food, thus forcing males to shift from female defence to territorial defence. Under these circumstances male kin-based alliances would be a major advantage. This pattern is found in the common chimpanzee. 


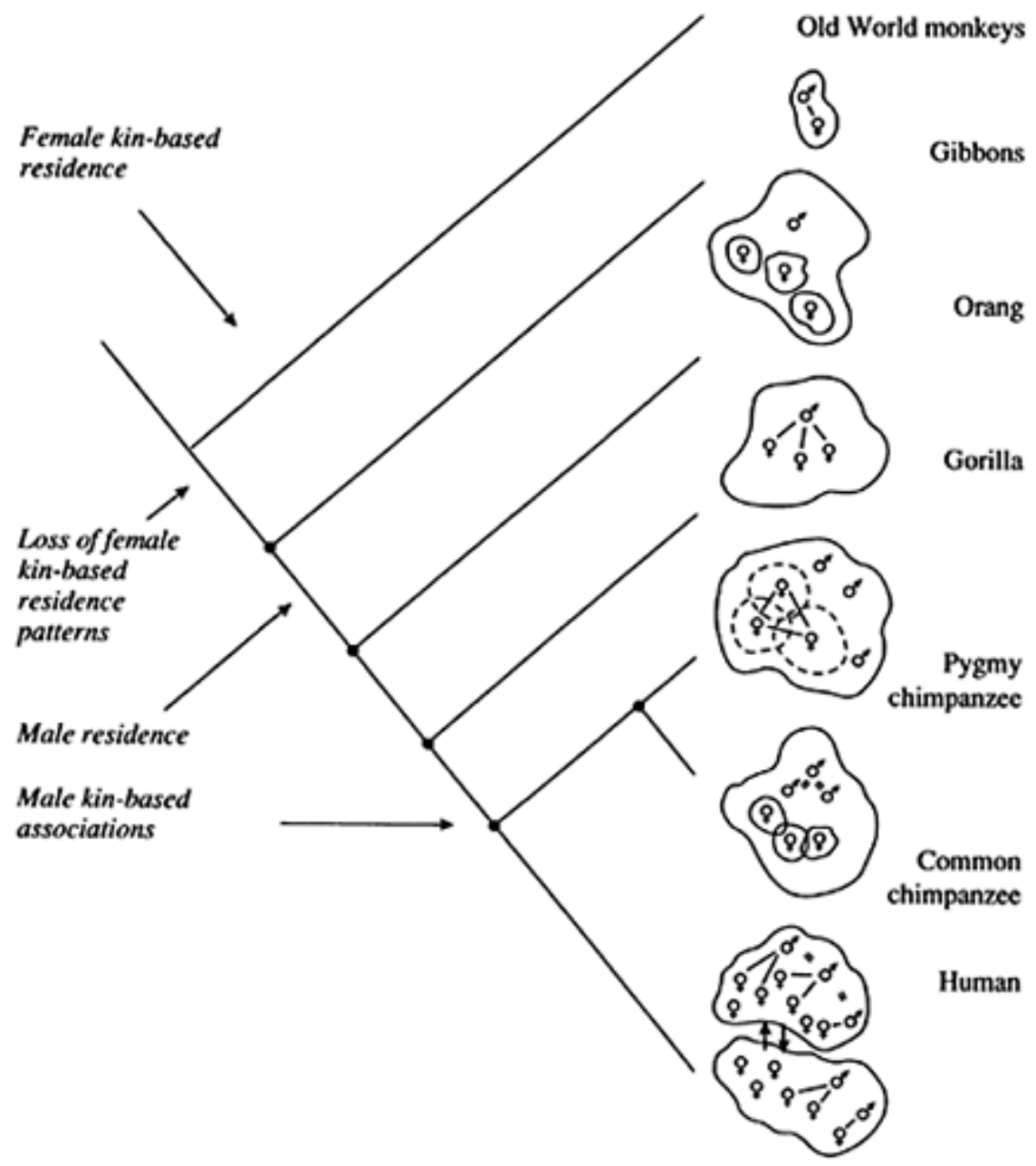

Figure 1.3 A cladistic phylogeny of catarrhine social systems. Female kinbased residence is considered to be the ancestral catarrhine system, which is lost in the hominoid clade. Male residence and male kinbonding becomes established in the African ape/hominid clade

It can be argued that the shift from female to male kin-based systems is the result of increasing body size leading to greater longevity, which in turn led to longer male tenure in monogamous or polygynous situations (Clutton-Brock 1989). Under these conditions the probability of males being able to disperse and find mates would be greatly reduced, resulting in greater benefits arising from remaining with their kin. Once established, the advantages of switching back to a female kin-based system are likely to be greatly reduced, and the number of state shifts required increased. Thus one can relatively simply express the routes that would need to be taken, and the ecological and social 
consequences of these state shifts (see also Foley 1989, Foley and Lee 1989, Lee, in press).

The key inference to be drawn is that the distribution of social states among the living catarrhines provides us with a strong indication concerning the ancestral social organization of the first hominids. The best evidence suggests that chimpanzees are the sister clade of the hominids (see Figure 1.3). This means first that hominids, as hominoids, come from a lineage in which female residence is absent or rare. Furthermore, there is within the 'trends' of hominoid evolution from lesser apes to African apes a tendency to observe increased male residence and kin-based organization. The conclusion that should perhaps be drawn is that the phylogenetic context for the origins of the patterns of social behaviour to be found among modern humans is one where any descent and alliance groupings are more likely to be male-based than female-based.

Clearly a number of caveats should be borne in mind when considering this model. One of these is that living species are not necessarily a good basis for inferring the events of the past. However, it should be noted that it is not individual species that are being used to determine the ancestral state (in the form of an analogue model), but the overall pattern of variability. The second qualification is that while this may well have been the ancestral state of the first hominids, we know that there is subsequently a very high level of evolutionary diversity to be found among the hominids of the early Pleistocene, and consequently a considerable range of derived social patterns should be expected.

\section{ECOLOGICAL CONTEXT OF HUMAN SOCIALITY}

The phylogenetic context for human social evolution described above might be taken to imply two things; first, that there is an inner drive to social evolution, and that once set on a particular path evolution will continue along it. The second is that phylogenetic heritage or historical factors are the primary determinants of human social evolution. However, as stated above (see p. 54), it is not solely the ancestral state that determines evolutionary change, but the interaction of a particular state with novel selective conditions. To understand the direction human social evolution has taken it is necessary to examine the ecological context in which it has occurred.

It is widely accepted among evolutionary biologists that there is a primary relationship between the evolution of more complex sociality and the evolution of larger brain size. This relationship is thought to hold across the primates as a whole, among other groups of mammals, and would be a strong theory that could account for the rapid and marked encephalization found in hominids and the high level of sociality that occurs in modern humans. Quantification and elaboration of this model has been provided by Dunbar (1992, Aiello and Dunbar 1993, and see this volume, chapter 15). He has shown that neocortex ratio (the ratio of the neocortex, the part of the brain responsible for higher cognitive functions, to the size of the brain as a whole) correlates strongly with group size among anthropoid primates. According to this model there is a reciprocal relationship in which social group size is constrained by the level of intelligence of the species, and in which the demands of sociality drives up the size of the neocortex. The evolution of higher intelligence is therefore, as Humphrey (1976) originally argued, a function of 
sociality.

Again, this model might be seen to imply a directionality and inherent drive to social evolution, with social complexity and intelligence driving each other upwards. In order to see the proper context in which this may have occurred in human evolution it is necessary to ask a different question - why has this spiralling process not occurred in other species? Answering this question provides us with a more complete understanding of the context in which human social evolution has occurred.

Sociality, as we have seen (see p. 48) is quite rare in the animal kingdom. While there are many benefits that accrue from social and group living (increased anti-predator vigilance, enhanced searching abilities, increased scope for kin-selection, territorial defence, etc.), there are costs as well (Standen and Foley 1989). Increased conspicuousness to predators, increased risk of infection and increased competition for food are all likely to occur with group living (Lee, in press). For some species these costs are too high to establish maintained sociality, and it is only when the benefits exceed the costs that stable sociality will evolve. The conditions under which this will occur are relatively rare, and relate to ecology.

Social groups can occur and be large when resources are clumped in large patches or where they are very uniformly and evenly spread across a landscape, allowing for a number of individuals to jointly exploit the resources. The important element is that the resources are extensive enough to be shared between a number of conspecifics without a significant reduction in individual intake. Furthermore, when high-quality food occurs in large patches, groups of related females may form both to exploit and to defend the resource (Wrangham 1980). Under such conditions, groups of related females thus suffer a smaller individual cost from the partitioning of resources amongst kin (reduced over the actual costs by the degree of relatedness), while gaining the considerable advantage of being able to control the resource against females who are not co-resident or related. Clearly, co-feeding need not lead to sociality, but in the presence of others who are in competition for that same resource, individuals who maintain sociality will gain an advantage.

Alternatively, if there is little or no significant advantage to attempting to control large patches of resources, females are less likely to aggregate. If the resources are widely enough spread, then the males can group females, who will tolerate co-feeding as there are few costs, and distinct advantages from staying with one or more males. Once male co-residence develops on top of some level of female co-residence, then there is the potential for the development of male kin distribution.

Thus, the distribution and quality of resources influences both the size and nature of the social groups that can form. Once social groups do form, then sociality will itself become a factor in determining further patterns of social evolution, but nonetheless the evolution of sociality is predicated upon both the initial and the sustaining ecological conditions.

There are many occasions when resources do not allow for continuous shared exploitation, but do facilitate concentrations of individuals either in localities or through time. In such animals, the resource base sets the initial limitations to the consistency and size of aggregations, while other considerations such as control of mates or rearing strategies may come into play. These species tend to have same-sex and opposite-sex 
distributions more related to reproductive strategies than to foraging or competitive strategies, as in the male kin-bonded groups. The point remains that the ecological conditions either enable or constrain the options for individual distributions with respect to conspecifics. For humans to develop such extreme social complexity implies that ecological strategies pursued by hominids were associated with environments that simultaneously constrained the formation of female-kin groups, while nonetheless enabling the formation of many other types of social systems.

There is thus a reciprocal relationship between sociality and ecological conditions in the same way that there is a relationship between sociality and brain size or intelligence. A further dimension can be added when we consider the remaining relationship, that between intelligence and ecology.

A number of authors (Clutton-Brock and Harvey 1980, Milton 1988, Gibson 1986) have argued that an interaction occurs between ecological variables and brain size as a measure of intelligence. It has been shown that both absolute and relative brain size increases with such variables as home range size and food quality (Figure 1.4). Two explanations for this pattern have been proposed. One, that exploitation of complex food types (evasive prey, seasonal resources, patchily distributed resources or rare food types) demand a greater level of skill than easily obtainable foods. Forest

\section{Taxonomic group}

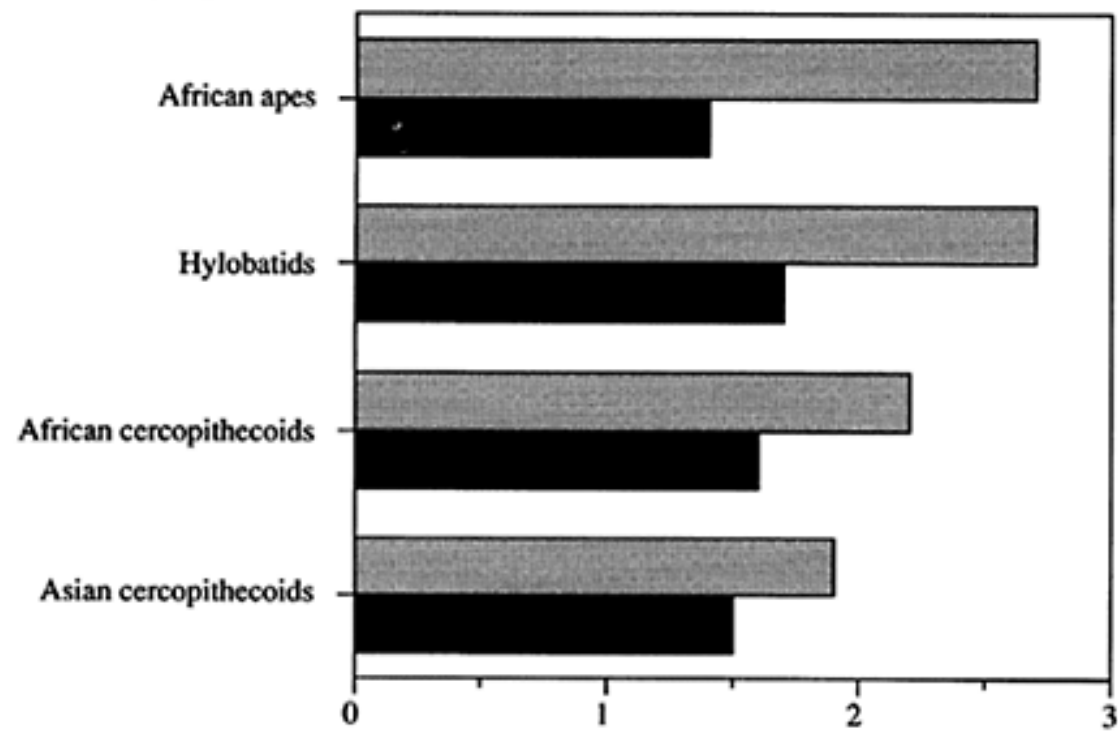

Encephalization quoticnt

\section{High quality diet $\square$ Low quality diet}

Figure 1.4 Relationship between Encephalization Quotient (EQ) and dietary quality for a sample of catarrhine primates. For each of the major clades the highest and lowest quality feeders were selected and their EQs compared. In each case the species with the better quality diet 
dwelling frugivorous primates would therefore be expected to have larger brains than folivores as their food type is less abundant. Alternatively, it has been argued that large brains are both metabolically expensive and isometrically related to metabolic rate. Animals with larger brains must therefore have a high quality and stable resource base.

In considering social evolution, therefore, we have a complex triangle of relationships. Sociality is clearly a major factor in driving the evolution of larger brains in primate and human evolution. However, the need to live in social groups is dependent upon the costs and benefits involved, and these are dependent upon the distribution of resources and the foraging strategies employed. Larger brains in turn require high levels of energy input, which means that their evolution can only occur under specific conditions of a high quality foraging strategy Intelligence and sociality in turn both strongly influence the way in which resources can be exploited (Figure 1.5).

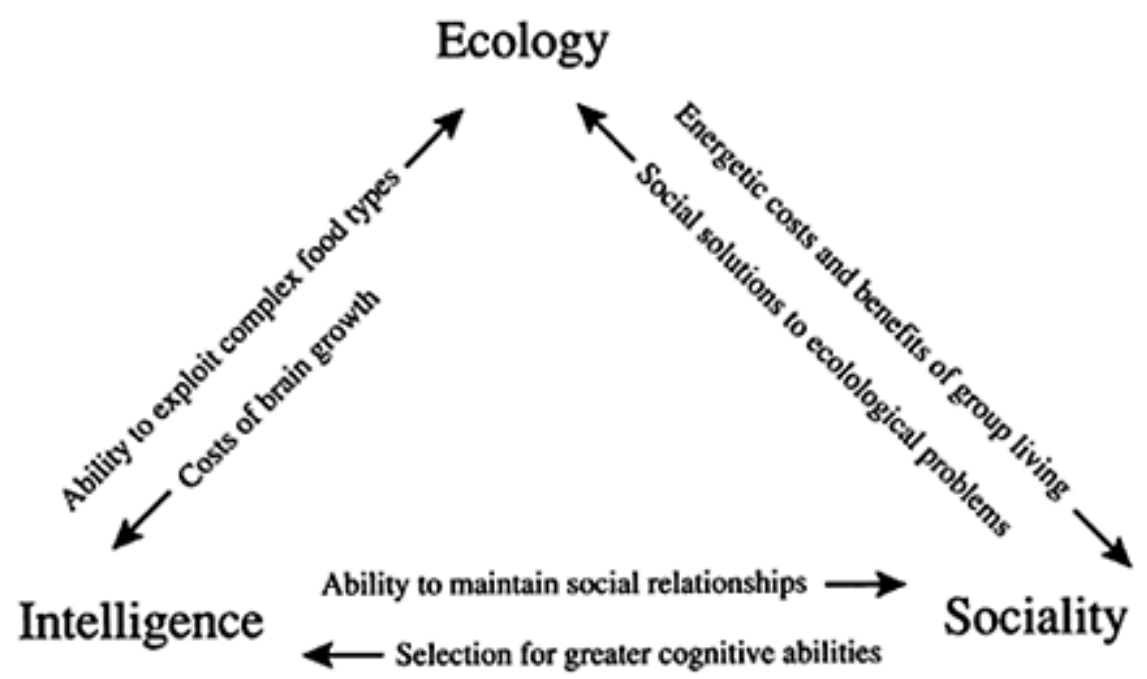

Figure 1.5 The nature of the interactions between ecological factors, sociality and intelligence

\section{LIFE-HISTORY THEORY AND A TENTATIVE TIMESCALE FOR HUMAN SOCIAL EVOLUTION}

Two useful conclusions can be drawn from this discussion of the phylogenetic and ecological context in which human sociality evolved. First, that human sociality is not the de novo origin of social behaviour, but is the addition of novel features onto existing strategies. This implies that any study of human social evolution should address the sequence and timing of the major changes that have occurred. Second, that sociality is not divorced from other biological elements, so that any consideration of social evolution should also address shifts in life-history strategy. This chapter will conclude with a brief 
discussion of these two problems.

Hominids diverged from the other African apes in the later Miocene (9-6 Myr), during a period of increasing climatic instability and aridity, and with an environmental shift away from forests towards more mosaic woodland and grassland. The ancestral conditions for the African hominoids can be surmised. In fully forested conditions there would have been a tendency towards closed, single male groups as is found today among gorillas. Resources would have been abundant and distributed in large and uniform patches. Females would have been able to survive within small home ranges, and in turn single males would be able to defend groups of females. In areas that were drier and as forests became less continuous and were replaced by woodland and savannah, the resources would become more dispersed and less uniformly distributed. Females would have to forage more widely, and males would thus no longer be able to employ a strategy of female defence. As is found among the common chimpanzee, larger communities would occur, with a breakdown of the classic harem system. With this females would no longer be attached to individual males, and related males would have to coexist to establish and maintain a territory. Thus it is in the late Miocene that male residence, female dispersal, male kin-bonding and larger communities became established as the basic social organization of the clade leading to both chimpanzees and humans.

During the Pliocene conditions in Eastern Africa became even drier and more seasonal. The effect of these new ecological conditions on the emerging hominid lineage can be seen in the establishment of bipedalism and other morphological changes, but there is increasing acceptance that for the most part the australopithecines are best thought of as bipedal apes rather than as anything very close to modern humans (Andrews and Martin 1991). There has, for example, at this stage been no significant increase in brain size. The same may well be true of their social organization which was most likely similar to that of the common chimpanzee. The main predicted effect would be a decrease in community size and an increase in community area-an effect that can be seen in chimpanzees across an environmental gradient (Foley 1993). However, caution should be exercised in this interpretation as among (mostly cercopithecoid) terrestrial and savannah primates there is a tendency towards larger group size, as a response either to the patchy resource distribution or to a higher incidence of predators.

The key change that needs to be incorporated into this model of social evolution is the change in the relationships between males and females. It can be argued that until this point - the 'australopithecine grade' - the only changes that have occurred lie within the potential range of variation for African apes, essentially variants on the theme of male kin-bonded groups. Patrilineal affinal kinship relationships may almost be said to be African ape plesiomorphies stretching back to the Miocene rather than unique human traits. In contrast, close and long-term bonding between males and females, with prolonged parental care and investment by both sexes, is unique to the human lineage. It is the timing of this that requires close examination.

Under what conditions should the bonding of males and females become more prolonged? Such bonding - and it should be noted that this is not necessarily implying exclusive monogamous bonds, but can apply equally to polygyny - is normally seen as evidence for increased parental, especially paternal, investment or effort. Male reproductive success is better served by close attention to the survivorship of putative 
offspring than in pursuing a more philandering strategy. This implies, as Lovejoy (1981) has pointed out, that infant survivorship is at stake and critical to male reproductive success. This led him to consider monogamy and the evolution of higher reproductive rates as critical. Two other factors seem more plausible. The first is that there may be a greater incidence of infanticide, and it may be the case that males are investing more to reduce the risk of infanticide by strange males. With the proposed model of a male kinbonded social system this may seem unlikely, although the possibility of inter-group encounters cannot be excluded.

Alternatively, paternal investment might be increased if the infant survivorship became increasingly dependent upon the quality of resources, and male protection or provisioning could offset the risks. The key question becomes under what conditions might this occur and how does any such change relate to the timing of human evolutionary changes.

Apart from the shift to bipedalism the other major change in hominid evolution is increased brain size. Rather than being a hominid trait this is in fact unique to the genus Homo and occurs predominantly in the last million years (i.e. the last 20 per cent of the hominid fossil record) (Foley 1992). Above it was argued that increased brain size imposes major metabolic costs on both mother and infant, and requires a stable and high quality resource base. In ecological terms this means that encephalization is dependent upon high quality resource availability, and without this it is unlikely that the extremely costly infants could be sustained. It is in this context that changes in male-female relationships should have occurred, with both males and females investing more in individual offspring.

In terms of the timing of these events a number of points can be made. First, the first significant increase in brain size in the hominid lineage occurs around 2.0 Myr, at about the time there is evidence for greater levels of meat-eating. With the establishment of Homo erectus there seems to be little doubt that some hominids were effective foragers for meat. Meat, it should be noted, constitutes a highly nutritious resource, especially in the seasonally variable African savannah environment. Second, models estimating the energetic costs of various levels of encephalization have shown that unless there are major changes in the rates of growth involved, the mother of an offspring with a far smaller brain than a modern human infant would face significantly greater costs (Foley and Lee 1991). In other words, changes in life-history parameters such as growth rates are integrally related to the brain size of the hominids. This implies that there is a close relationship between changing foraging strategies, life-history strategies and social behaviour in hominid evolution.

Attempting to pinpoint exactly when the critical changes occurred is problematic. Initial increases in male-female bonding may have occurred with the beginnings of brain size expansion, but the most significant effects are unlikely to occur until later. Modelling suggests that a cranial capacity of about $1,000 \mathrm{cc}$ is the critical threshold where human growth rates are required (Foley and Lee 1991). This occurs in 'late Homo erectus', or around half a million years ago. Aiello and Dunbar (1993) have also argued that this is both the brain size when primate systems of social relationship maintenance would break down, and when there is also evidence for an acceleration in the rate of encephalization. Furthermore, there is dental evidence that the rate of growth in early Homo erectus $(>1.0$ Myr) is still much faster than that of modern humans, but that by later H. erectus the life- 
history changes were already in place (Smith 1989, 1992, Foley and Lee 1991). All this points to the possibility that while some changes in sociality were gradual throughout the Pleistocene, the late middle Pleistocene represents a significant point of inflection both biologically and behaviourally. This fact should be remembered in considerations of the context in which the so-called 'human revolution' of the Upper Palaeolithic occurred.

\section{CONCLUSIONS}

This brief consideration of the possible timing of the key events in human social evolution has led to the suggestion that some human social features are best considered as deep plesiomorphies from our hominoid ancestry, but that others may have occurred much later in conjunction with shifts in both ecology and life-history strategies. Affinal kinship structures are likely to lie further back in the hominid ancestry (and indeed be ultimately hominoid) than male-female mating strategies. In summary, we have argued that social evolution in humans has been predicated upon both ecological conditions and life-history parameters, rather than being a process divorced from other evolutionary changes. The fact that sociality is a major force in evolution does not mean that the potential for social behaviour and the actual social strategies pursued are not subject to biological constraints. As we have seen, the elaboration of social behaviour that is so characteristic of our own species is deeply dependent upon the phylogenetic and ecological context in which we evolved. Human social behaviour, far from being a tapestry of infinite variety, lies within the finite social space that constrains all mammals, and has not been built from new but is a continuation of the highly successful primate strategy of solving ecological problems by social means.

\section{REFERENCES}

Aiello, L.C. and Dunbar, R.I.M. (1993) 'Neocortex size, group size and the evolution of language.' Current Anthropology 34:184-193.

Andrews, P. and Martin, L. (1991) 'Hominoid dietary evolution.' Philosophical Transactions of the Royal Society (London) Series B 334:199-209.

Clutton-Brock, T.H. (1989) 'Female transfer, male tenure and inbreeding avoidance in social mammals.' Nature 337:70-72.

Clutton-Brock, T.H. and Harvey, P.H. (1980) 'Primates, brains and ecology.' Journal of Zoology 190:309-323.

Dunbar, R.I.M. (1992) 'Neocortex size as a constraint on group size in primates.' Journal of Human Evolution 22:469-493.

Emlen, S.T. and Oring, L.W. (1977) 'Ecology, sexual selection and the evolution of mating systems.' Science 197:215-223.

Foley, R.A. (1989) 'The evolution of hominid social behaviour.' In V.Standen and R.A.Foley (eds) Comparative Socioecology, pp. 473-494. Oxford: Blackwell Scientific Publications.

Foley, R.A. (1991) 'How useful is the culture concept in early human studies?' In R.A.Foley (ed.) The Origins of Human Behaviour, pp. 25-28. London: Unwin Hyman. Foley, R.A. (1992) 'Evolutionary ecology of fossil hominids.' In E.A.Smith and 
B.Winterhalder (eds) Evolutionary Ecology and Human Behavior, pp. 131-164. Chicago: Aldine de Gruyter.

Foley, R.A. (1993) 'The influence of seasonality on hominid evolution.' In S.J. Ulijaszek and S.Strickland (eds) Seasonality and Human Ecology, pp. 17-37. Cambridge: Cambridge University Press.

Foley, R.A. and Lee, P.C. (1989) 'Finite social space, evolutionary pathways and reconstructing hominid behavior.' Science 243:901-906.

Foley, R.A. and Lee, P.C. (1991) 'Ecology and energetics of encephalization in hominid evolution.' Philosophical Transactions of the Royal Society (London) Series B 334:223-232.

Ghiglieri, M.P. (1987) 'Sociobiology of the great apes and the hominid ancestor.' Journal of Human Evolution 16:319-357.

Gibson, K.R. (1986) 'Cognition, brain size and the extraction of embedded food resources.' In J.Else and P.C.Lee (eds) Primate Ontogeny, Cognition and Social Behaviour, pp. 93-105. Cambridge: Cambridge University Press.

Goodall, J. (1986) The Chimpanzees of Gombe. Cambridge, MA: Belknap Press.

Harcourt, A. and Stewart, K. (1987) 'The influence of help in contests on dominance rank in primates: hints from gorillas.' Animal Behaviour 35:182-190.

Hinde, R.A. (1976) 'Interactions, relationships and social structure.' Man 11: 1-17.

Hinde, R.A. (1983) Primate Social Relationships: An Integrated Approach. Oxford: Blackwell.

Humphrey, N.K. (1976) 'The social function of intellect.' In P.P.G.Bateson and R.A.Hinde (eds) Growing Points in Ethology, pp. 303-317. Cambridge: Cambridge University Press.

Ingold, T. (1990) 'An anthropologist looks at biology.' Man 25:208-229.

Kano, T. (1992) The Last Ape: Pygmy Chimpanzee Behaviour and Ecology. Palo Alto: Stanford University Press.

Lee, P.C. (1992) 'Biology and behaviour in human evolution.' Cambridge Archaeological Journal 1:207-226.

Lee, P.C. (in press) 'Social structure and evolution.' In P.Slater and T.Halliday (eds) Behaviour and Evolution. Cambridge: Cambridge University Press.

Lovejoy, C.O. (1981) 'The origin of man.' Science 211:341-50.

McGrew, W.C. (1992) Chimpanzee Material Culture. Cambridge: Cambridge University Press.

Milton, K. (1988) 'Foraging behaviour and the evolution of primate intelligence.' In R.Byrne and A.Whiten (eds) Machiavellian Intelligence, pp. 285-305. Oxford: Clarendon.

Smith, B.H. (1989) 'Dental development as a measure of life history in primates.' Evolution 43:683-688.

Smith, B.H. (1992) 'Life history and the evolution of human maturation.' Evolutionary Anthropology 1:134-142.

Standen, V. and Foley, R.A. (eds) (1989) Comparative Socioecology: The Behavioural Ecology of Humans and Other Mammals. Special Publication of the British Ecological Society No. 8. Oxford: Blackwell Scientific.

Struhsaker, T. and Leland, L. (1987) 'Colobines: infanticide by adult males.' In B.Smuts, D.L.Cheney, R.M.Seyfarth, R.W.Wrangham and T.Struhsaker (eds) Primate Societies, pp. 83-97. Chicago: Chicago University Press.

Trivers, R.L. (1972) 'Parental investment and sexual selection.' In B.Campbell (ed.) Sexual Selection and the Descent of Man, pp. 139-179. Chicago: Aldine. 
Finite social space and the evolution of human social behaviour 59

Wrangham, R.W. (1980) 'An ecological model of female-bonded primate groups.' Behaviour 75:262-300.

Wrangham, R.W. (1987) 'The significance of African apes for reconstructing human evolution.' In W.G.Kinzey (ed.) The Evolution of Human Behavior: Primate Models, pp. 28-47. Albany: SUNY Press. 